\title{
Carcinoma urotelial ureteral bilateral sincrónico
}

\author{
I. Bravo Fernández, J.V. García Cardoso, G. Pérez Ortiz, J. Gómez, C. González Enguita, \\ R. Vela Navarrete
}

Cátedra y Servicio de Urología. Fundación Jiménez Díaz-UTE. Universidad Autónoma de Madrid.

Actas Urol Esp 2006; 30(3): 335-339

\section{RESUMEN}

CARCINOMA UROTELIAL URETERAL BILATERAL SINCRÓNICO

Objetivo: Presentar el caso de un tumor urotelial ureteral bilateral sincrónico sin afectación vesical. Métodos: Diagnóstico y tratamiento de un caso de tumor urotelial bilateral sincrónico.

Conclusiones: El carcinoma urotelial bilateral sincrónico sin afectación vesical es una entidad rara (1-5\% de todos los tumores uroteliales) donde la cirugía radical sigue siendo el tratamiento curativo de elección, aunque el mejor conocimiento de esta patología hace que hoy en día se pueda realizar tratamiento conservador a un grupo seleccionado de pacientes con tasa de similar supervivencia a los que se le indica tratamiento radical.

Palabras clave: Tracto urinario superior. Carcinoma urotelial sincrónico. Tratamiento.

\section{ABSTRACT}

SYNCHRONOUS BILATERAL UROTHELIAL CARCINOMA OF THE UPPER URINARY TRACT Objective: Report a case of a synchronous bilateral urothelial carcinoma of the upper urinary tract without bladder affectation.

Methods: We describe the diagnosis and treatment of a case of a bilateral upper urothelial carcinoma.

Conclusions: Synchronous bilateral urithelial carcinoma of the upper urinary tract without bladder affectation in an unusual occurance (1-5\% of urothelial tumors) and radical surgery continues to be the treatment of choice, although it is possible to take a less aggressive approuch with selected groups of patient, in which we can achiese a survival rate similar to that which we obtain with radical surgery.

Keywords: Upper urinary tract. Urothelial carcinoma. Treatment.

$\mathrm{L}$ os tumores del tracto urinario superior son una rara entidad, suponen entre el 1- 5\% de todos los tumores uroteliales. Pueden aparecer de forma sincrónica o metacrónica. La localización a nivel de pelvis renal es cuatro veces más frecuente que la ureteral. El uréter distal está afecto en el $70-75 \%$ de los casos.

Se ha considerado la cirugía radical como tratamiento curativo de elección, aunque el mejor conocimiento hace que pueda realizarse tratamiento conservador en un grupo seleccionado de pacientes, con igual tasa de supervivencia.
A continuación presentamos el caso de un varón de 68 años de edad con carcinoma urotelial ureteral bilateral sincrónico sin afectación vesical.

\section{CASO CLÍNICO}

Se trata de varón de 68 años de edad remitido desde su ambulatorio por presentar uropatía obstructiva bilateral, en urografía intravenosa, realizada para el estudio de cuadro de hematuria.

Como antecedentes presentaba dislipemia, hiperplasia benigna de próstata, infarto agudo de miocardio, diabetes mellitus no insulino dependiente. 
Refería tres episodios de hematuria en diciembre de 2001, agosto de 2002 y enero del 2003 autolimitados, asintomáticos y sin coágulos.

A la exploración física se observaba paciente con buen estado general, abdomen globuloso, blando, depresible, sin masas ni megalias, no doloroso a la palpación. Tacto rectal se palpaba próstata compatible con hiperplasia benigna grado III/IV.

\section{Pruebas Analiticas Complementarias}

Hemograma: Parámetros dentro de los límites de la normalidad.

Bioquímica de sangre: glucosa $145 \mathrm{mg} / \mathrm{dl}$. El resto de parámetros estaba dentro de los límites de la normalidad. PSA 9,09 PSAl 1,43 índice 15,73

Estudio sistemático de orina: PH6,5, densidad1.016, negativo para proteínas, cuerpos cetónicos, bilirrubina, nitritos, urobilinógeno y leucocitos, $0,50 \mathrm{~g} / 1$ de glucosa y $50 \mu \mathrm{l}$ de sangre.

Sedimento de orina: 1-2 hematíes por campo. Citología benigna (negativa para malignidad).

\section{Técnicas de Imagen}

Radiografía simple de abdomen: Calcificaciones en pelvis menor de posible origen vascular. Buena distribución de gas intestinal. Líneas del psoas visibles.

UIV (Fig. la y 1b): Riñones de tamaño, forma y localización dentro de los límites de la normalidad. Eliminación renoureteral bilateral y simétrica con dilatación del colector renal izquierdo, tercio superior-medio de uréter izquierdo con imagen de disminución del calibre a nivel de región pélvica que puede estar en relación con cálculo radiotransparente. Colector renal derecho no muestra alteración observándose, a nivel del uréter pelviano, defecto de replección en posible relación con cálculo radiotransparente o coágulo. En la cistografía se observa vejiga irregular, sugestiva de trabeculaciones con marcada impronta prostática.

Pielografia percutánea izquierda (Fig. 2): Hidronefrosis con paso de contraste hasta vejiga, observándose defecto de replección a nivel de uréter pelviano, a unos $5 \mathrm{~cm}$ de vejiga.
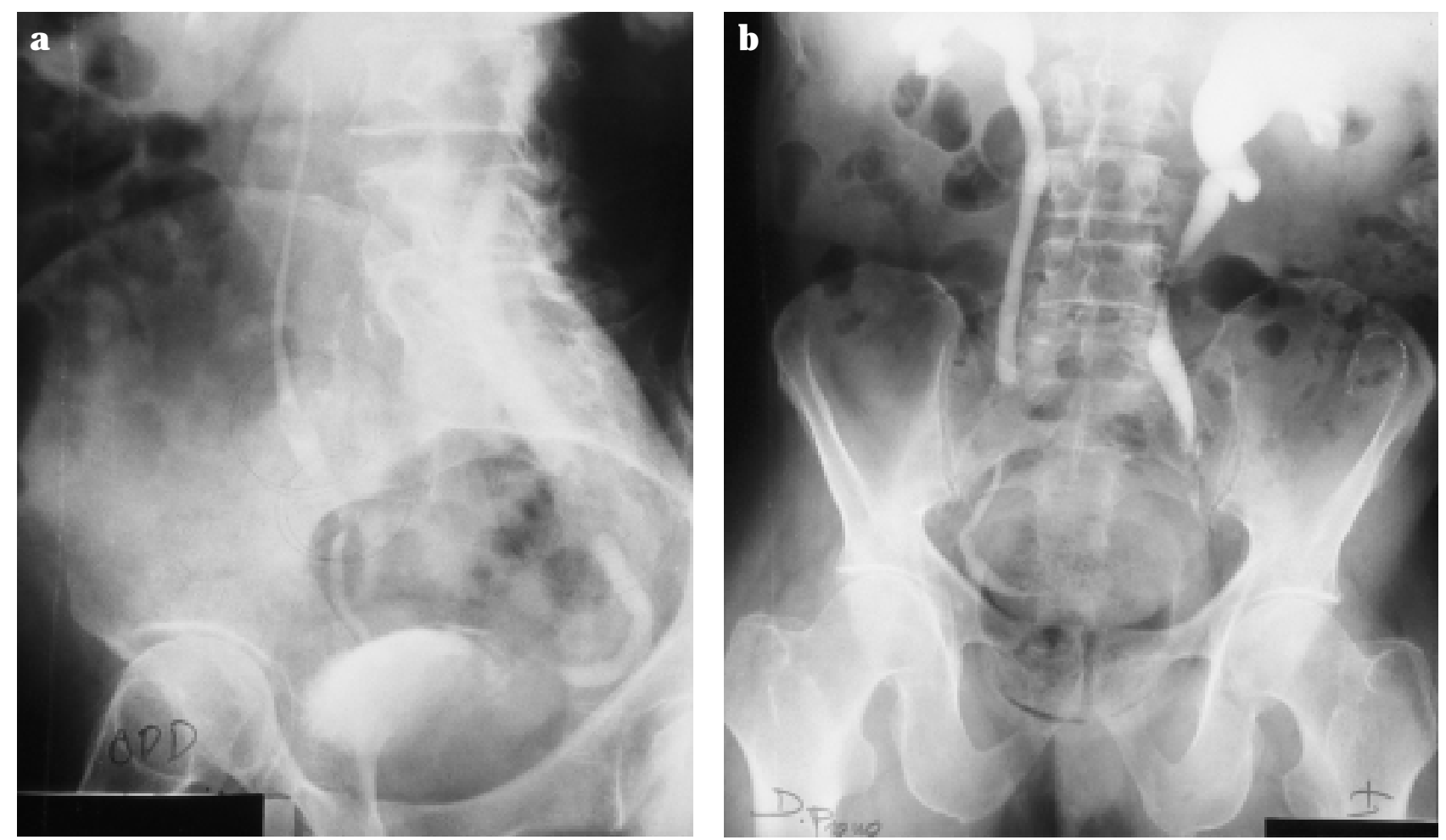

FIGURA 1a y 1b: UIV Uropatia obstructiva secundaria a defecto de replección en ambos uréteres Iliacos. Proyecciones oblicua (1a) y anteroposterior (1b). 


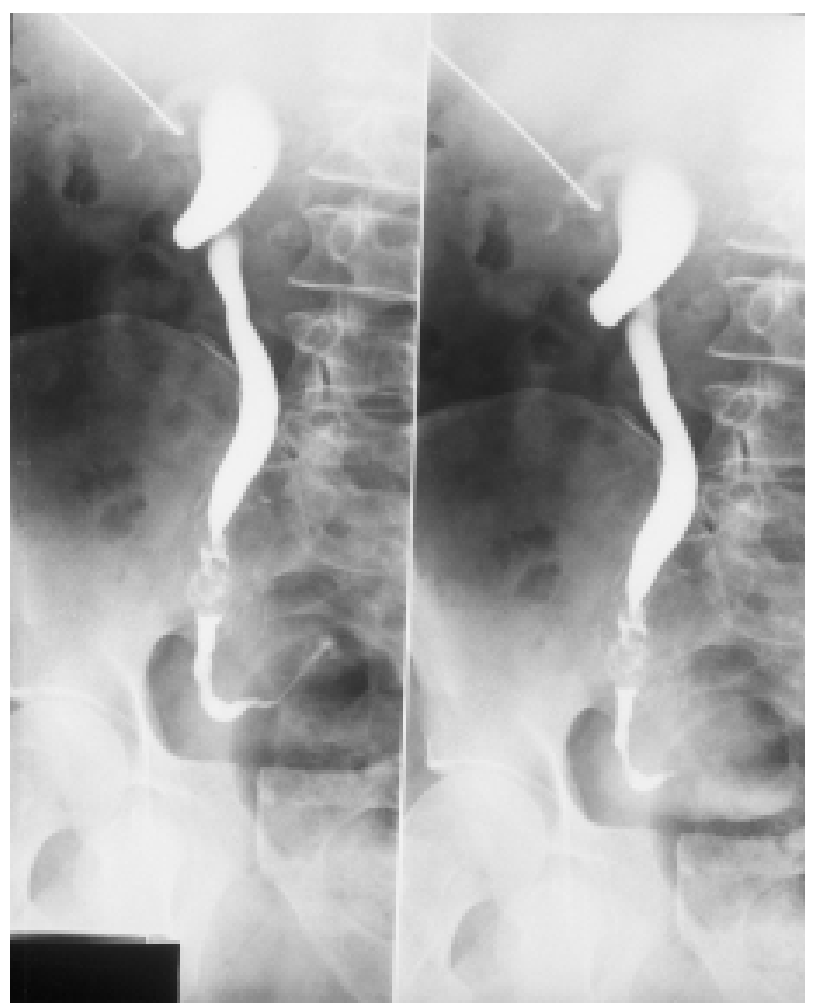

FIGURA 2: Pielografia anterógrada izquierda Visualizándose defecto de replección de aproximadamente $3 \mathrm{~cm}$ de diámetro sugerente de neoformación urotelial.

TAC abdómino-pélvico (Fig. 3): Moderada hidronefrosis bilateral $(26 \mathrm{~cm}$ la pelvis renal derecha y aproximadamente $29 \mathrm{~cm}$ la izquierda, en el eje transversal). En ambos uréteres distales, aproximadamente a $5 \mathrm{~cm}$ de distancia de la vejiga urinaria, se observa contenido intraureteral, de densidad de partes blandas, sugestivo de urotelioma bilateral, ocupando prácticamente toda la luz de los uréteres, aunque sin ocluirlos totalmente, y ocupando una longitud aproximada de $2 \mathrm{~cm}$. Resto de estructuras abdomino-pélvicas no se observan alteraciones aparentes.

$\mathrm{RM}$ pélvica: Dilatación de riñón izquierdo sin poder identificar causa exacta de la estenosis.

\section{Tratamiento}

Mediante laparotomía media transperitoneal se exponen trayectos ureterales desde zona lumbar hasta yuxtavesical objetivándose dos masas intraureterales, móviles, de aproximadamente $2 \mathrm{~cm}$ de longitud, con tejidos periureterales de aspecto normal. Se decide ureterotomía bilateral longitudinal apreciando dos neoforma-

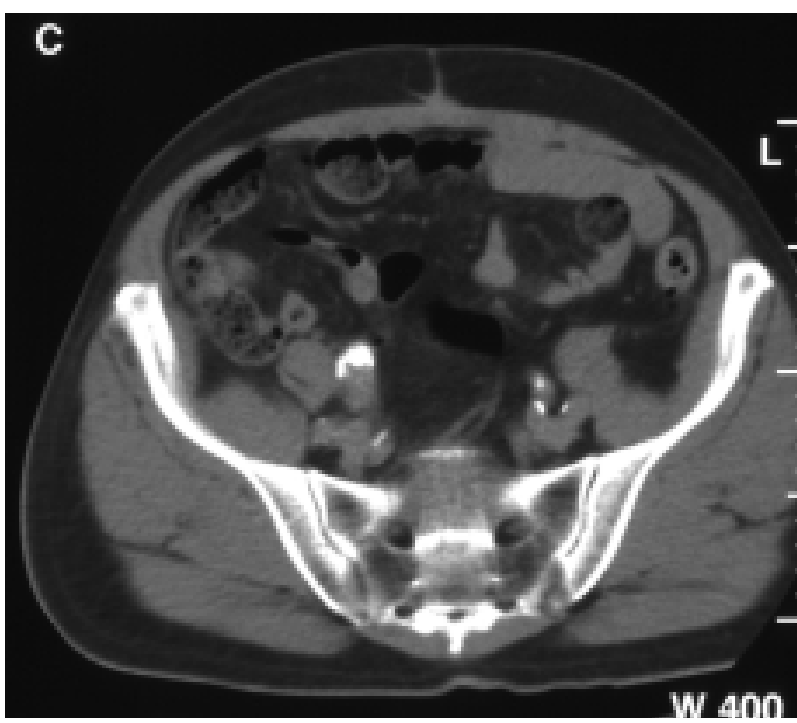

FIGURA 3: TAC Tumoración que ocupa la luz ureteral derecha con engrosamiento de su pared. Engrosamiento de pared ureteral izquierda.

ciones papilares de base de implantación pediculada decidiéndose su extirpación. Colocación de pig-tail y cierre de uretrotomia.

\section{Anatomía Patológica}

Descripción macroscópica: Fragmento irregular, blanco-rosado de $3 \times 2,5 \times 1,2 \mathrm{~cm}$ el izquierdo y $3 \times 2,5 \times 1,2 \mathrm{~cm}$ el derecho. Al corte se reconoce eje central en ambos.

Descripción microscópica: Los tumores de ambos uréteres presentan un aspecto similar. Corresponden a carcinoma urotelial papilar, de bajo grado citológico, con ejes conectivo-vasculares tapizados por múltiples hileras de urotelio con leve pleomorfismo y excepcionales mitosis. Ambos presentan eje centrado músculo-vascular, libre de infiltración tumoral, aunque en zonas el tumor contacta con el músculo aparentemente sin corion subyacente. La base de resección con signos de fulguración, libre. A nivel del tumor izquierdo en uno de sus bordes, existe proliferación papilar, mientras en el opuesto se conserva urotelio sin displasia significativa. En la del derecho parecen libres ambos bordes, aunque en uno de ellos existe resto de tumor papilar fulgurado adosado.

\section{Diagnóstico Final}

Carcinoma urotelial papilar G1 estadio de difícil valoración, probablemente $\mathrm{T} 1$. 


\section{Evolución Clínica}

El paciente es dado de alta tras 14 días de ingreso, durante el cual presentó cuadro febril secundario a neumonía basal derecha que respondió satisfactoriamente al tratamiento antibiótico. También presentó cuadro de ileo paralítico que se recuperó tras la aplicación de medidas conservadoras.

Acudió 10 días después para retirada de pigtail sin presentar ninguna complicación.

En su primera revisión tras la cirugía, tres meses después, el paciente se encuentra asintomático desde el punto de vista urológico presentando urografía intravenosa normal.

\section{DISCUSIÓN}

Los tumores del tracto urinario superior son una entidad rara, suponen alrededor del 5\% de los tumores uroteliales. La presentación bilateral y sincrónica alcanza según estadísticas el 1-5\%, pudiendo llegar hasta el 5\% en regiones endémicas de nefropatía balcánica ${ }^{1}$. La frecuencia de aparición se incrementa con la edad, siendo una rareza en niños. En el adulto más del 95\% son de estirpe epitelial, excepcional los de estirpe mesenquimatosa ${ }^{2}$. Dentro de los factores etiológicos destacamos edad, sexo, raza y habito tabáquico como más importantes sin olvidar analgésicos, ciclofosfamida, café, exposición a carcinógenos profesionales, antecedentes de infección y litiasis del tracto urinario.

El signo clínico más frecuente es la hematuria, con o sin dolor acompañante. El dolor aparece por distensión pielocalicial secundaria a obstrucción, consecuencia del crecimiento tumoral o por coágulos.

La citología de orina es el principal método de diagnóstico inicial, así como para el seguimiento en estos pacientes. Se trata de un método fiable de diagnóstico, teniendo en los "in situ" y G3, una sensibilidad del 90\% y una especificidad entre el 98-100\%. En los tumores de bajo grado, pese a su baja sensibilidad, es también útil como método diagnóstico y de seguimiento, sobre todo en muestras obtenidas selectivamente mediante cateterismo ${ }^{3}$.

El diagnóstico por imagen se realiza mediante ecografía, urografía intravenosa, pielografía anterógrada o retrógrada, TAC.
El tracto urinario superior tiene pequeñas pero importantes diferencias estructurales, anatómicas e histológicas, si lo comparamos con la vejiga, como son la estrechez de la luz uretral, su menor espesor, entre otras. Esto condiciona una serie de peculiaridades clínico-patológicas. Además, la rica vascularizacion y drenaje linfático de las vías altas urinarias facilitan la diseminación metastásica retroperitoneal. Macroscópicamente son tumores muy variables. Se puede encontrar desde una pequeña neoformación, papiloma, hasta el tumor rígido, ulcerado, necrótico $^{2}$.

La nefroureterectomia con resección de rodete vesical ha sido considerado el tratamiento de elección en pacientes con tumor tracto urinario superior $^{4-6}$. Se indicaba tratamiento conservador (preservar parénquima renal y urotelio) en situaciones de bilateralidad, monorrenos e insuficiencia renal. Pero el mejor conocimiento de la evolución y factores pronósticos de estos tumores ha permitido ampliar las indicaciones del tratamiento conservador ${ }^{5}$.

Los principales inconvenientes de este tipo de cirugía son la recidiva y la progresión. El porcentaje de recurrencia es del $15-45 \%$ según series, siendo mayor tras cirugía endoscópica que tras cirugía abierta ${ }^{5}$. Se sabe que son factores de alto riesgo de recidiva los tumores a nivel de pelvis renal, tumor asociado a tumor vesical, citología positiva y multifocalidad, y factores de mal pronóstico la insuficiencia renal, tumor infiltrante y G3 ${ }^{4,5}$. Por tanto, el grupo de pacientes que podrían beneficiarse de tratamiento conservador serían aquellos con tumor único, de bajo grado y pequeño tamaño ${ }^{4-6}$. La administración de BCG o Mitomicina C in situ disminuye las recurrencias ${ }^{5}$.

Sobre los pacientes que se realiza tratamiento conservador se debe realizar un estrecho seguimiento debido al alto riesgo de recidiva (que en algunos casos puede ser asintomática) y proliferación ${ }^{4-6}$.

En conclusión, podemos decir que el tratamiento radical de estos tumores se lleva a cabo en aquellos tumores infiltrantes y/o de alto grado reservando tratamiento conservador para los tumores superficiales y bien diferenciados. El seguimiento de estos pacientes debe ser un seguimiento individualizado, independientemente del 
tipo de cirugía que se haya llevado a cabo, además de ser un seguimiento muy estrecho durante largo tiempo por el alto porcentaje de recidiva.

\section{REFERENCIAS}

1. Holmang S, Johansson SL. Synchronous bilateral ureteral and renal pelvis carcinomas. Cancer 2004;101(4)741-747.

2. García González R, Cuesta Roca C, García Navas R, González Peramato P. Anatomía patológica de los tumores del tracto urinario alto (uréter, pelvis y cálices renales). Arch Esp Urol 2004;57(3):241-250.

3. González Peramato P, Jiménez Heffernan JA, García González R, García Navas R. Citología de orina en el diagnóstico del tumor urotelial del tracto urinario superior. Arch Esp Urol 2004;57(3):227-238.

4. Iborra I, Solsona, E, Casanova J, Ricós JV, Rubio J, Climent MA. Conservative elective treatment of upper urinary tract tumors: A multivariate analiysis of prognostic factors for recurrence and progression. J Urol 2003;169:82-85.
5. Muñoz Vélez D, Llullul R, Hidalgo Pardo F, Riera Mari V, Mus Maellu A, Gutierrez Sanz-Gadea C, et al. Tumores del tracto urinario superior: resultados del tratamiento y seguimiento. Arch Esp Urol 1999;52(4):333-341.

6. Portillo Martín JA, Rado Velásquez MA, Gutierrez Baños JL, Corres Gómez MA, Hernández Rodríguez R, del Valle Shaan $\mathrm{JL}$, et al. Tumores del urotelio superior. Actas Urol Esp 2004;28(1)7-12.

Dra. Inmaculada Bravo Fernández

Fundación Jiménez Díaz-UTE

Avda Reyes Católicos, 2

28040 Madrid

(Trabajo recibido el 21 de junio de 2005) 